\title{
E-Learning Framework for Learning Disabled Children
}

\author{
Zainab Pirani \\ M.H.Saboo Siddik College of \\ Engineering. \\ Mumbai, India
}

\author{
Vasiqullah Molvizadah \\ M.H.Saboo Siddik College of \\ Engineering. \\ Mumbai, India
}
Sasikumar M., PhD.
CDAC Kharghar
Navimumbai, India

\author{
Mohammad Abbas \\ Sayyed \\ Thakur College of Management \\ Studies and Research \\ Mumbai, India
}

\begin{abstract}
The purpose of this paper is to provide a an e-learning framework for understanding functional limitations related to learning Disability (LD) and to identify various learning principles and dimensions that can be used to overcome limitations and enhance functional capacities in home, school and college settings. The focus of the research is limited to the academic disorders associated with LD and will not address the physical and sensory limitations that often accompany LD.
\end{abstract}

\section{General Terms}

E-Learning, Human Computer Interaction, Disabilities

\section{Keywords}

E-learning Framework, Learning Disability, Learning Dimensions, special needs

\section{LEARNING DISABILITY}

According to the National Centre for Learning Disability, LD is a neurological disorder that affects the brain's ability to receive, process, store and respond to information [1]. These skills are essential for success at school and college for coping with academic life in general. LD is not an indicative of intelligence level. Rather, children with LD have trouble performing specific types of skills or completing tasks if left to figure things out by themselves or if taught in conventional ways. LD should not be confused with Learning Difficulty. LD are lifelong and do not respond readily to any intervention. But learning difficulty can be improved by making use of various education interventions.

\subsection{Types of Learning Disabilities}

LD is a broad term. There are many different kinds of LD. Most often they fall into three broad categories and students affected by LD have more than one kind. Each of these categories includes a number of more specific disorders as shown in the figure 1 and explained in sections below [2]:

\subsubsection{Speech and Language Disorder}

Speech and language problems are often the earliest indicators of a learning disability. People with developmental speech and language disorders have difficulty producing speech sounds, using spoken language to communicate, or understanding what other people say. Depending on the problem, the specific diagnosis may be categorized as following disorders

\subsubsection{Articulation disorder}

Students with this disorder may have trouble controlling their rate of speech. Or they may lag behind classmates in learning to make speech sounds. For example, A 6-year old who still says "wippon" instead of "rippon" and "thnake" for "snake."

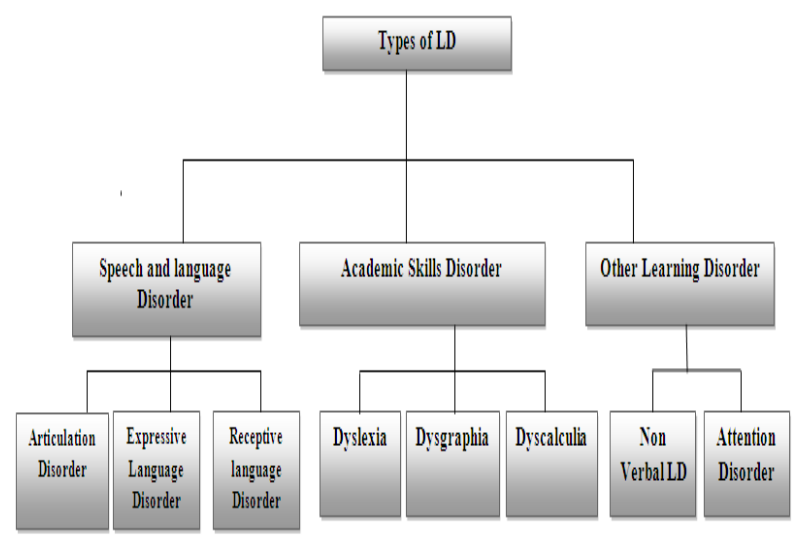

Fig 1: Types of LD

\subsubsection{Expressive language disorder}

Students with this disorder have problems expressing themselves in speech. For example, a 4-year-old who speaks only in two-word phrases and a 6-year-old who can't answer simple questions.

\subsubsection{Receptive language disorder}

Students with this disorder have trouble understanding certain aspects of speech. It's as if their brains are set to a different frequency and the reception is poor. For example, a 4-year-old who doesn't respond to his name, a 6-year-old who hands you a mat when you asked for a bat.

\subsubsection{Academic Skills Disorder}

Students with academic skills disorders struggles a lot $\mathrm{n}$ area of reading, writing and arithmetic skills. The diagnosis in this category include: 


\subsubsection{Dyslexia-Reading Disorder}

Students with this disorder have problems in encoding and decoding words. They have very slow reading rate and tend to forget which line was read and repeat the same line again. For example, a 8-year-old who can't identify the word "battle" "caterpillar".

\subsubsection{Dysgraphia-Writing Disorder}

Students with this disorder may have problems in writing, spelling, organization of ideas, and composition. For example, a 6-year-old gets pain in hand muscles if he writes one paragraph consisting of 30 words.

\subsubsection{Dyscalculia-Arithmetic Disorder}

Students with this disorder cause such difficulties as learning math concepts, arranging numbers, doing money transactions For example, a 6 -year-old faces problem to solve simple sums like $9+5=14$ or $8 \times 5=40$.

\subsubsection{Other Learning Disorder}

Students with this disorder include certain coordination disorders and learning handicaps. The diagnoses in this category include:

\subsubsection{Non verbal learning disability}

Students with this disorder often manifest in motor clumsiness, poor visual-spatial skills, problematic social relationships, difficulty with math, and poor organizational skills. For example, a 4-year-old who have problem in writing, running, jumping etc because the brain is unable to communicate with the necessary limbs to complete the desired $\operatorname{task}[4]$.

\subsubsection{Attention disorder}

A behavioral condition called Attention Deficit Hyperactivity Disorder (ADHD) is often associated with LD because students with ADHD have a hard time focusing enough to learn and are often easily distracted. For example, a 3-yearold who has ADHD act impulsively, running into traffic, toppling desks, blurt out answers, interrupt and can't sit still.

\subsection{Difficulty in Education with Learning Disabilities}

Children with LD can face unique challenges in their educational life. The kinds and severity of problems faced may vary from individual to individual. They may do well in some areas, but very poorly in others. They may learn what is seen, but not what is heard; they may remember by writing, but not by reciting orally and so on. Some of the common difficulties faced by then are listed below [3] [4]:

- inconsistent school performance

- difficulty remembering today what was learned yesterday, but may know it tomorrow

- $\quad$ short attention span (restless, easily distracted)

- $\quad$ letter and number reversals (sees "b" for "d" or "p", "6" for "9", "pots" for "stop" or "post")

- $\quad$ poor reading (below age and grade level)
- frequent confusion about directions and time (rightleft, up-down, yesterday-tomorrow)

- deficits in study skills, such as test preparation, note taking, and listening comprehension

- personal disorganization (difficulty in following simple directions/schedules; has trouble organizing, planning, and making best use of time; frequent loss or misplacement of homework, schoolbooks, or other items)

- failure on written tests but high scores on oral exams (or vice versa)

With such types of problems, LD students avoid going to schools and are often years behind their classmates which leads to failure in their academic life.

This paper is organized as follows: Technological intervention in education for LD is covered in Section 2. The proposed learning environment and its various components are discussed in Section 3. Section 4 concludes the paper.

\section{TECHNOLOGICAL INTERVENTION IN EDUCATION FOR LD}

To overcome the above discussed problems complete support is required from parents, teachers and technology. An extensive literature survey was conducted to understand the need of LD student with respect to the technological intervention in education, which can be used as an active part of the learning process to extend the children's abilities. With technology becoming more accessible, it has become a critical learning tool that is being encouraged frequently in schools. The use of technology can be a helpful tool for teachers and students to focus on ability rather than disability [5]. Also there are two main areas where technological intervention has direct impact on LD students in their academic life as shown in the table 1

Table 1. Technological Intervention

\begin{tabular}{|c|l|}
\hline Areas & \multicolumn{1}{|c|}{ Technological Intervention } \\
\hline $\begin{array}{c}\text { Individualized } \\
\text { Learning }\end{array}$ & $\begin{array}{l}\text { Usages of images, audio, video } \\
\text { components specific to individual } \\
\text { disabilities }\end{array}$ \\
\hline $\begin{array}{l}\text { Remedial } \\
\text { Learning }\end{array}$ & $\begin{array}{l}\text { Practice session for remembering every } \\
\text { lesson module. }\end{array}$ \\
\hline
\end{tabular}

First area enriches teaching by rich multimedia explorations and provides individualized learning i.e. depending upon the requirement of individual LD student, appropriate teaching strategies embedded with various multimedia elements is delivered to the student which increases their academic performances. Second area facilitates remediation through practice and guided instruction for struggling LD learners. The guided instruction helps LD students to maintain their moral which is drastically affected due to constant failure. With guided instruction, the hances of failure become negligible and they develop interest in their academic career. [6].We also see that e-learning is playing a significant role in education and its importance is increasing day by day. Students use online content in addition to textbooks and the classes are held on the web [7]. So the aim is to combine all the above three areas in a platform which could be accessible 
for LD students as well as available all time which is explained in the next section.

\section{PROPOSED E-LEARNING FRAMEWORK FOR LD}

The proposed e-learning framework provides a road map that can be taken to improve the educational performance of LD students by connecting them to relevant processes and practices. It provides a foundation for transforming education in the form required by the LD students.

\subsection{Phases}

The proposed framework is designed to work on the following four phases as shown in the figure 2. Each phase is built on set of principles, refer section 3.2. And each principle is accompanied with various dimensions required for any type of e-learning framework, refer section 3.3.

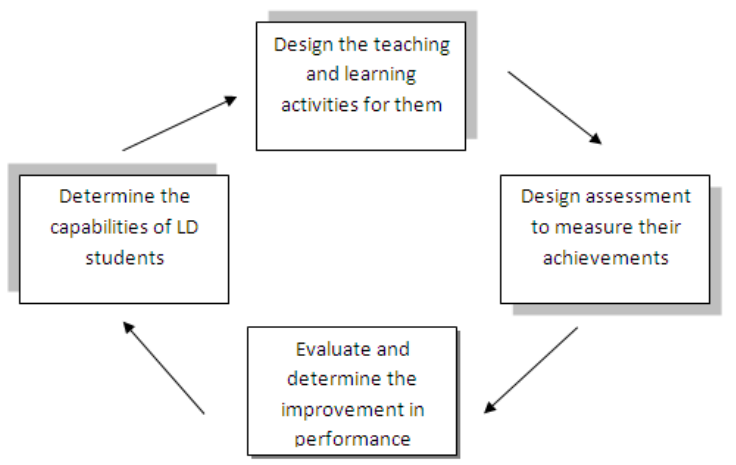

Fig 2: Four Phases of the proposed framework

\subsubsection{Phase 1-Determine the capabilities of LD student}

Many LD students have difficulty in understanding and using a language which causes problems in reading, writing, and comprehension and have poor understanding of mathematical concepts as explained in section 1.2. So it is necessary to identify individual capability to cope up with the existing academic problems. This is done by conducting various LD diagnostic test to identify the exact level of each and every individual LD student.

\subsubsection{Phase 2 - Design the teaching and learning strategies for them}

Depending upon the individual capabilities of LD student, various instructional methods have to be designed. The scenario is same as that of classroom. But the only difference is that the teacher is not available to see the response of the LD student. So designing various strategies is one of the most important and time consuming task.

\subsubsection{Phase 3 - Design assessment to measure their achievements}

Once the LD students have completed their activity, it is necessary to check whether they have understood it or not. So now they have to undertake an assessment session which will actually measure their achievement level.

\subsubsection{Phase 4 - Evaluate and determine the improvement in performance}

Based on the assessment level, the actual result is evaluated with the prior result to identify its performance and determine whether that LD student is improving or not. If the evaluation result is improving, same teaching and learning activities are continued. If the evaluation is same as previous or detoriating then it becomes necessary that learning and teaching strategies should be modified.

Thus, we see that every phase plays a vital role in the elearning framework design.

\subsection{Principles}

The principles defined in the e-learning framework, lay the foundation in determining strategies for all the phases and evaluate them to know the improvement in the performance. Every phase of the proposed e-learning framework is built on set of principles which is explained as follows:

\subsubsection{Phase 1}

The aim of phase 1 is to determine the capabilities of the LD students and it deals with the following set of principles:

\subsubsection{Level of understanding}

Every LD student has its own way of understanding or learning things. There are three categories based on the level of understanding of LD students. The first category is called as audio level of understanding. In this the LD students can understand and remember easily if audio is involved in the teaching process. The second category is called as visual level of understanding in which the LD student learns by actually seeing the teaching concept and then he understands and remembers. The third category of LD student deals is multisensory level of understanding, in which both the above styles are strong enough to grasp the teaching content.

\subsubsection{Grasping power}

Each and every LD student will have its own level of grasping power. It varies from slow to fast. LD student with slow grasping power have to take lots of effort to understand a simple learning concept. Medium grasping power does not require extra effort to understand the task whereas quick grasping power has tremendous effect on their learning skills.

\subsubsection{Phase 2}

The aim of phase 2 is to design teaching and learning strategies for the LD students and it deals with the following set of principles.

\subsubsection{Flexibility in learning and teaching}

As nature of LD students is varying in nature, it becomes necessary that some sort of flexibility should be available in learning and teaching them. Also as the age of LD students grows, their learning requirements and teaching requirements changes.

\subsubsection{Medium of teaching}

Based on type of level of understanding and grasping power, the medium of teaching will vary. For eg., if the level of understanding is audio and grasping power is slow, then the LD student will be provided with slow speed lecture in audio 
format with frequent pauses and a chance to forward and do reverse to the audio lecture.

\subsubsection{Phase 3}

The aim of phase 3 is to design assessment to measure the LD students achievements and it deals with the following set of principles.

\subsubsection{Standards for assessment}

It is necessary to set a standard to assess LD student assessment which includes diagnostic, process, and outcome assessment-which are integral parts of the learning process.

\subsubsection{Mode of assessment}

As the LD student face lots of problem in writing exam papers. It becomes necessary to identify the mode in which they can give their exam and we identify their mode of assessment. Most common mode of assessment used for LD students is making use of TTS (Text to speech) converter to understand the written content. And STT (Speech to text) converter to avoid spelling mistakes, grammatical mistakes etc.

\subsubsection{Phase 4}

The aim of phase 4 is to evaluate and determine improvement in the performance of the LD students and it deals with the following set of principles.

\subsubsection{Level of evaluation}

Based on the severity of learning disability, the evaluation is done at three levels. The first level deals with mild type of LD in which the evaluation is in its strict mode i.e. no extra consideration is done while evaluating the test or exam. The second level deals with moderate type of LD, in which evaluation takes into consideration many factors like avoidance of spelling mistakes, time taken etc. The last level deals with severe type of LD, in which evaluation level is in very light mode. Here the aim is to avoid all the silly and careless mistakes and just focus on the approach of the student in order to do a particular task.

\subsubsection{Motivational influences}

LD students face failures many times in their academic career, so it becomes very necessary that they get some sort of motivation. The motivational influences have direct impact on the performance of the LD student. With various motivation techniques, the memory, perception and learning process of LD students can be improved.

\subsection{Dimensions}

Every phase of the e-learning framework is based on set of dimensions. This dimension provides us systematic way to understand factors that make up the e-learning framework which are as follows:

\subsubsection{Classification}

The classification dimension examines various categories of LD students, based on which the teaching strategies will be decided.

\subsubsection{Pedagogical}

The pedagogical dimension refers to classification dimension and depending upon that categorization, various analysis on teaching strategies are considered

\subsubsection{Technological}

The technological dimension refers to the infrastructural requirements in the e-learning framework, which includes hardware and software requirements.

\subsubsection{Content}

The content dimension refers to accessibility guideline that is to be followed to generate appropriate materials for $\mathrm{LD}$ students.

\subsubsection{Presentation}

The presentation dimension includes set of methods to deliver the content in the form required by the LD students.

\subsubsection{Interface design}

The interface design dimension refers to the final screen that LD student will view. It deals with look and feel of the learning programs.

\subsubsection{Evaluation}

The evaluation dimension provides feedback of the LD students with respect to their performance in the e-learning environment.

Thus based on above dimensions, e-learning framework can be designed to overcome the difficulties associated with LD students.

\section{CONCLUSION}

Software developers, designers and researchers have been looking towards various e-learning frameworks for solutions to help and educate students with LD. But none are available to fulfill the needs of LD students. So with the proposed elearning framework specifically designed for LD students, the aim is to overcome their learning difficulties from academically point of view. This framework will be further enhanced to cover all the physical and sensory limitations accompanied by LD students.

\section{REFERENCES}

[1] National Center for Learning Disabilities, 2006. [Online].Available http://ncld.org/LDInfoZone/InfoZoneFactSheet_LD.cfm

[2] Diagnostic Centre [Online]. Available http://www.wrongdiagnosis.com/l/learning_disabilities/s ubtypes.htm

[3] Learning Disabilities Online, 2010. Retrieved on $21^{\text {st }}$ May,2011 from http://www.ldonline.org/ldbasics/signs

[4] Health Care \& Diagnostic Centre, 2011[Online]. Retrieved on $2^{\text {nd }}$ May, 2011 from http://www.wrongdiagnosis.com///learning_disabilities/s ubtypes.htm

[5] Rhodes, J. \& Milby, T. M. (2007). Teacher-Created Electronic Books: Integrating Technology to Support Readers with Disabilitites. The Reading Teacher, 61(3), 255-259. 
International Journal of Computer Applications (0975 - 8887)

Volume 63-No.19, February 2013

[6] Kimberly L. Keith. 2006, Assistive Technology for Students with Learning Disabilities, J. Reach learn. Res 3 1276-1282

[7] Beverly Park Woolf .2010, A Roadmap for Education Technology funded by the National Science Foundation \# 0637190, The Computing Community Consortium
(CCC), managed by the Computing Research Association (CRA) with a sub-award to Global Resources for Online Education, Beverly Park Woolf, P. I, pg 69. 